\title{
REVIEW
}

\section{Knowledge and Opportunities from the Plastisphere: A Prelude for the Search of Plastic Degrading Bacteria on Coastal Environments}

\section{Luis Felipe Avilés-Ramírez ${ }^{1}$ Joanna M. Ortiz-Alcantara ${ }^{2}$ Ma. Leticia Arena-Ortiz ${ }^{2 *}$}

1. Faculty of Sciences, National Autonomous University of Mexico (UNAM), Yucatán, Mexico

2. Laboratory of Ecogenomic Studies, Faculty of Sciences, National Autonomous University of Mexico (UNAM), Yucatán, Mexico

\begin{tabular}{l}
\hline ARTICLE INFO \\
\hline Article history \\
Received: 2 August 2021 \\
Accepted: 30 August 2021 \\
Published Online: 15 September 2021 \\
\hline Keywords: \\
Plastic biodegradation \\
Bacteria \\
Plastisphere \\
Bioremediation \\
Coastal environments
\end{tabular}

\section{Introduction}

\section{The plastic pollution problem}

Plastics are synthetic or semi-synthetic polymers mainly produced from petrochemicals, characterized by their high resistance/density relationship, their great thermal and electric isolation properties, as well as resistance to acids, alkalis and solvents ${ }^{[1]}$. These materials have applications in multiple industries and business sectors like trading, packing, building materials, medical and pharmaceutical uses, automotive industry, home appliances, agriculture and many mass production products for the every-

\begin{abstract}
Plastic pollution has become an urgent issue, since its invasion to every ecosystem has led to multiple impacts on the environment and human populations. Certain microbial strains and genera had shown the ability to biodegrade plastic sources under laboratory conditions. In this minireview, we collect and analyze scientific papers and reports of this microbial activity as we contextualize this information on the global plastic pollution problem, to provide an updated state of the art of plastic biodegradation with microbial agents. Along with a broad understanding of the general process of plastic biodegradation hosted by microorganisms. The contributions of this minireview come from the identification of research gaps, as well as proposals for new approaches. One of the main proposals focuses on coastal environments and in particular coastal wetlands as a great microbiome source with potential for plastic biodegradation, whether reported or undiscovered. Our final proposal consists of the application of this knowledge into technologic tools and strategies that have a remarkable impact on the battle against the plastic pollution problem.
\end{abstract}

day human consumption ${ }^{[2,3]}$.

These polymers pollute and invade a lot of environments due to the single-use and improper waste management. The main plastic types considered environmental pollutants are High-Density Polyethylene (HDPE), Low-Density Polyethylene (LDPE), Polyvinyl Chloride (PVC), Polystyrene (PS), Polypropylene (PP), Polyester (PES), Polyamide (PA) and Polyethylene terephthalate $(\mathrm{PET})^{[4]}$.

Plastic pollution has become one of the biggest environmental threats by invading every ecosystem. It has been reported the presence of plastic debris (microplastics)

*Corresponding Author:

Ma. Leticia Arena-Ortiz,

Laboratory of Ecogenomic Studies, Faculty of Sciences, National Autonomous University of Mexico (UNAM), Yucatán, Mexico; Email:leticia.arena@ciencias.unam.mx 
from deep-sea sediments ${ }^{[5]}$, to the highest areas of the mount Everest ${ }^{[6]}$. The presence of microplastics in the human placenta has been documented as well ${ }^{[7]}$.

Around 13 million tons of plastic have been thrown to oceans annually, besides the global annual production of 300 million tons of plastic debris ${ }^{[8]}$. In México, nearly 83,343 tons of trash are collected daily ${ }^{[9]}$ and in the municipal solid waste composition, plastic spans $10.9 \%{ }^{[10]}$.

Main consequences of this pollutant relay on contamination of water sources like rivers, lakes or oceans leading to the formation of continental sized trash patches in the middle of the Pacific Ocean ${ }^{[11]}$. Also, additives and other compounds present in plastics impact on coastal, marine and inland soils, damaging their physicochemical properties and fertility ${ }^{[12]}$.

Biotic impacts come from mutilation, intoxication and asphyxia of marine, coastal and terrestrial organisms ${ }^{[13]}$. Human population affectations include the intake of microplastic polluted food ${ }^{[14]}$ to neurotoxic damage from PAH's, Phthalates and PCB's ${ }^{[15]}$. Furthermore, plastic residues represent public health and economic hazards to human communities.

\section{Coastal Wetland Ecosystem Goods and Services}

Worldwide ecosystems shelter goods and services that provide human populations resources for their basic needs; these so-called ecosystem services and natural capital ${ }^{[16]}$ are in decay because of environmental issues such as plastic contamination. This situation is a threat to global human economics and welfare, ever since biodiversity is linked in so many complex ways to ecosystem functionalities and their output ecosystem services ${ }^{[17]}$.

Particularly, coastal wetlands provide human population wide and diverse goods and services due to the enormous biodiversity within these ecosystems. Some of these goods and services are categorized as ecosystem services framework ${ }^{[18]}$. Supporting ecosystem services include the primary production of microbiotic (bacteria, fungi, protozoea) and macrobiotic (migratory birds and commercial interest fish and crustaceans) life forms, soil formation and enrichment. Provisioning goods and services are represented by sources like fresh water, seafood, honey and woods. Regulating functions such as climate regulation by carbon dioxide sequestration and flood and storms mitigation. The Cultural category is covered as aesthetics, recreational, educative and spiritual values for native human population as well as foreign people through ecotourism ${ }^{[19]}$.

\section{Microbial Biodegradation of Plastics}

Environmental plastic pollution solutions have become an urgent subject and an interesting approach could be just below ourselves. Diverse microbes have shown the ability to degrade plastics; particularly bacterial strains isolated from different environments that have been studied and reported in diverse scientific publications. Some of these papers are reviewed in the present study.

Bacteria have been widely researched in plastic biodegradation matter, since they are easy for cultivation and isolation, and the facility for bioprospecting metabolic pathways, ecological functions and subproduct related information through metagenomic analysis and sequencing of the $16 \mathrm{~S}$ ribosomal gene ${ }^{[20,21]}$. In this review, scientific evidence of plastic biodegradation hosted by bacteria dates from at least $1991^{[22,23]}$. In general, research has focused on confirming and assessing plastic degradation by bacteria either through in vitro or in situ assays.

Depolymerization activity is usually measured with diverse methodology such as visual assessment through detecting roughening, cracking and biofilm formation ${ }^{[24]}$, clear-zone tests ${ }^{[25]}$, weight loss estimates in microbial exposure ${ }^{[26]}$, respiratory activity evaluation such as $\mathrm{CO}_{2}$ production or $\mathrm{O}_{2}$ consumption ${ }^{[27]}$. As well as detection of the activity of specific enzymes or byproducts of depolymerization activity ${ }^{[28,29]}$.

\section{Bacterial Biodegradation State of the Art}

In the present review, we summarize some bacterial strains and species that have the ability to biodegrade plastic, with complementary information about the habitat of the bacteria, type of plastic degraded, byproducts, enzyme and the bibliographic reference to the paper. This information is categorized and divided based on the type of environment where the bacteria were isolated.

Table 1 shows previous scientific research results from bacteria isolated from anthropic environments, such as municipal waste disposal sites, sewage water, industrial activated sludges or purchased pre-cultured strains from laboratories and microbial strains collections.

Aquatic environment native bacteria are represented in Table 2. Aquatic environments that hold bacterial sources are wide and diverse and some of the most studied environments are sea water and soil, abyssal water and soil, freshwater like rivers and lakes. Some of the least studied are coastal soil and water, highlighting coastal wetland ecosystems.

http://dx.doi.org/10.36956/sms.v3i2.432 
Sustainable Marine Structures | Volume 03 | Issue 02 | July 2021

Table 1. Municipal waste disposal sites/Other Human environments

\begin{tabular}{|c|c|c|c|c|c|}
\hline ID & Habitat & Plastic type degraded & $\begin{array}{l}\text { Degradation by- } \\
\text { products }\end{array}$ & Enzyme & Reference \\
\hline $\begin{array}{l}\text { Pseudomonas, Penicillium, } \\
\text { Rhodotorula, Hyalodendron }\end{array}$ & Landfill & $\begin{array}{c}\text { Low Density } \\
\text { Polyethylene (LDPE), } \\
\text { Polyurethane (PU), } \\
\text { Polyvinyl chloride (PVC) } \\
\text { Polyethylene glycol }\end{array}$ & - & $\begin{array}{c}\text { Alcano monoxigenase, } \\
\text { same as found } \\
\text { on hydrocarbon } \\
\text { biodegradation } \\
\text { (Seneviratne, 2006) }\end{array}$ & {$[26]$} \\
\hline Ideonella sakaiensis & $\begin{array}{l}\text { Sediment from PET- } \\
\text { recycling site. }\end{array}$ & $\begin{array}{l}\text { Polyethylene } \\
\text { terephthalate (PET) }\end{array}$ & $\begin{array}{l}\text { Terephthalic acid } \\
\text { (TPA) \& ethylene } \\
\text { glycol (EG) }\end{array}$ & $\begin{array}{c}\text { Mono(2-hydroxyethyl) } \\
\text { (MHET) terephthalic } \\
\text { acid }\end{array}$ & {$[30]$} \\
\hline Pseudomonas & Final waste deposition site & $\begin{array}{c}\text { Low Density } \\
\text { Polyethylene (LDPE) }\end{array}$ & - & - & {$[31]$} \\
\hline Comamonas acidovorans & City soil samples & $\begin{array}{l}\text { Polyester-type } \\
\text { polyurethanes }\end{array}$ & $\begin{array}{l}\text { Adipic acid and } \\
\text { diethylene glycol }\end{array}$ & - & {$[32]$} \\
\hline Pseudomonas aeruginosa & $\begin{array}{c}\text { Previously isolated } \\
\text { microorganisms } \\
\text { (Microteca/microlibrary) }\end{array}$ & $\begin{array}{c}\text { PET, PU, PP, ABS, } \\
\text { HDPE, PVC, ABS, PS }\end{array}$ & - & - & {$[24]$} \\
\hline Acidovorax delafieldii & City soil samples & $\begin{array}{c}\text { Poly(tetramethylene } \\
\text { succinate)-co- } \\
\text { (tetramethylene adipate) } \\
\text { (PBSA) }\end{array}$ & - & Lipase & {$[33]$} \\
\hline $\begin{array}{l}\text { Bacillus subtilis, Bacillus } \\
\text { cereus, Bacillus lentus, } \\
\text { Pseudomonas aeruginosa, } \\
\text { Staphylococcus aureus, } \\
\text { Klebsiella pneumoniae, } \\
\text { Streptococcus pyogenes, } \\
\text { Escherichia coli, Proteus } \\
\text { vulgaris, Micrococcus. }\end{array}$ & FADAMA soil & $\begin{array}{c}\text { Polythene plastic bags } \\
\text { and environmental plastic } \\
\text { materials }\end{array}$ & - & $\begin{array}{c}\text { Polyurethanases } \\
\text { (Koutny et al., 2006). }\end{array}$ & {$[34]$} \\
\hline Brevibacillus borstelensis & $\begin{array}{l}\text { Soil from Polyethylene } \\
\text { waste deposition site }\end{array}$ & $\begin{array}{l}\text { Branched low-density } \\
\text { polyethylene }\end{array}$ & - & - & {$[35]$} \\
\hline Rhodococcus ruber & $\begin{array}{c}\text { Sediments with } \\
\text { polyethylene debris from } \\
\text { agriculture use }\end{array}$ & Polyethylene & - & Esterases & {$[36]$} \\
\hline $\begin{array}{c}\text { Streptomyces viridosporus, } \\
\text { Streptomyces badius and } \\
\text { Streptomyces setonii }\end{array}$ & $\begin{array}{l}\text { Enzymes from cultured } S \text {. } \\
\text { viridosporus, } S \text { badius and } \\
\text { S setonii }\end{array}$ & $\begin{array}{c}\text { Starch-polyethylene } \\
\text { degradable plastic films }\end{array}$ & $\begin{array}{c}\text { Primary and } \\
\text { secondary alcohols }\end{array}$ & - & {$[23]$} \\
\hline $\begin{array}{c}\text { Pseudomonas putida (AJ) and } \\
\text { Ochrobactrum (TD) }\end{array}$ & $\begin{array}{c}\text { Dangerous waste disposal } \\
\text { site }\end{array}$ & Vinyl Chloride & - & - & {$[37]$} \\
\hline Pseudomonas fluorescens & $\begin{array}{c}\text { Naval Research Laboratory, } \\
\text { Wahington D.C. }\end{array}$ & Polyester polyurethane & - & $\begin{array}{l}\text { Enzyme with protease } \\
\text { activity }\end{array}$ & {$[38]$} \\
\hline Thermomonospora fusca & Compost from green waste & $\begin{array}{l}\text { Aliphatic-Aromatic } \\
\text { Copolyesters } \\
\text { (synthesized from } \\
\text { 1,4-butanediol, adipic } \\
\text { acid, and terephthalic } \\
\text { acid (BTA)) }\end{array}$ & - & - & {$[39]$} \\
\hline $\begin{array}{c}\text { Schlegelella } \\
\text { thermodepolymerans and } \\
\text { Pseudomonas indica (K2) }\end{array}$ & Activated sludge & $\begin{array}{c}\text { Poly }(3- \\
\text { hydroxybutyrate-co-3- } \\
\text { mercaptopropionate). } \\
\text { [poly(3HB-co- } \\
\text { 3MP)] }\end{array}$ & $\begin{array}{l}\text { 3HB Oligomer } \\
\text { linked as thioester }\end{array}$ & $\begin{array}{c}\text { Poly }(3- \\
\text { hydroxybutyrate })(3 \mathrm{HB}) \\
\text { depolymerase }\end{array}$ & {$[40]$} \\
\hline
\end{tabular}




\begin{tabular}{|c|c|c|c|c|c|}
\hline ID & Habitat & Plastic type degraded & $\begin{array}{l}\text { Degradation by- } \\
\text { products }\end{array}$ & Enzyme & Reference \\
\hline $\begin{array}{l}\text { Clostridium botulinum and } \\
\text { Clostridium acetobutylicum }\end{array}$ & $\begin{array}{l}\text { Sewage slugde and methane } \\
\text { sludge }\end{array}$ & $\begin{array}{l}\text { Poly(b-hydroxybutyrate) } \\
\text { (PHB), poly(b- } \\
\text { hydroxybutyrate- } \\
\text { co- } 11.6 \%-b- \\
\text { hydroxyvalerate) } \\
\text { (PHBV) and the synthetic } \\
\text { polyester poly(o- } \\
\text { caprolactone) (PCL) }\end{array}$ & 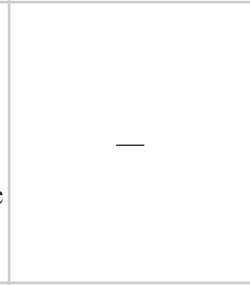 & PCL depolymerizing & [25] \\
\hline $\begin{array}{c}\text { Bacillus amylolyticus, } \\
\text { Bacillus firmus, Bacillus } \\
\text { subtilis, Pseudomonas putida, } \\
\text { Pseudomonas fluorescens }\end{array}$ & $\begin{array}{l}\text { Municipal solid waste from } \\
\text { compost plant }\end{array}$ & Polyethylene bags & - & - & [41] \\
\hline $\begin{array}{c}\text { Bacillus cereus, } B . \\
\text { megaterium, } \text { B. subtilis, } \\
\text { Brevibacillus borstelensis }\end{array}$ & Culture fields & Polyethylene & $\begin{array}{l}\text { Hydrocarbons } \\
\text { (saturated and } \\
\text { unsaturated) and } \\
\text { alcohols of higher } \\
\text { molecular weight }\end{array}$ & - & [42] \\
\hline Rhodococcus rhodoshrous & $\begin{array}{c}\text { Purchased isolates from } \\
\text { American Type Culture } \\
\text { Collection }\end{array}$ & $\begin{array}{c}\text { High density } \\
\text { polyethylene } \\
\text { (HDPE), Low density } \\
\text { polyethylene (LDPE) } \\
\text { and Linear low-density } \\
\text { polyethylene (LLDPE) } \\
\text { films with balanced } \\
\text { content of antioxidants } \\
\text { and pro-oxidants } \\
\text { (manganese+iron or } \\
\text { manganese+iron+cobalt) }\end{array}$ & - & - & [43] \\
\hline $\begin{array}{l}\text { Actinomadura sp. S14, } \\
\text { Actinomadura sp. TF1, } \\
\text { Streptomyces sp. APL3 y } \\
\text { Laceyella } \mathrm{sp} \text {. TP4 }\end{array}$ & Compost soil & $\begin{array}{l}\text { Polyester biodegradable } \\
\text { plastics; polylactic acid } \\
\text { (PLA), polycaprolactone } \\
\text { (PCL), poly-(butylene } \\
\text { succinate) (PBS) \& } \\
\text { polybutylene succinate- } \\
\text { co-adipate (PBSA) }\end{array}$ & - & $\begin{array}{c}\text { S14, TF1 \& TP4 } \\
\text { produced PLA and } \\
\text { PBSA depolymerase } \\
\left(50^{\circ} \mathrm{C}\right), \text { APL3 }\left(40^{\circ} \mathrm{C}\right) \text {. } \\
\text { Actinomadura sp. S14 } \\
\text { (PCL depolymerase) } \\
\text { Actinomadura sp.TF1 } \\
\text { (PLA depolymerase), } \\
\text { Streptomyces sp. APL3 } \\
\text { (PBS depolymerase), } \\
\text { Laceyella sp.TP4 } \\
\text { (PBSA depolymerase) }\end{array}$ & [44] \\
\hline $\begin{array}{l}\text { Paenibacillus amylolyticus } \\
\text { TB-13 (Bacillus amylolyticus) }\end{array}$ & $\begin{array}{l}\text { Sediment samples from } \\
\text { multiple sites }\end{array}$ & $\begin{array}{c}\text { Poly(lactic acid), } \\
\text { poly(butylene succinate), } \\
\text { poly(butylene } \\
\text { succinateco-adipate), } \\
\text { poly(caprolactone) and } \\
\text { poly(ethylene succinate) }\end{array}$ & - & Proteases and esterases & [45] \\
\hline Streptomyces sp. AF-111 & $\begin{array}{c}\text { Sewage sludge from } \\
\text { Treatment Plant Rawalpindi } \\
\text { Pakistan }\end{array}$ & $\begin{array}{l}\text { Poly(3-hydroxybutyrate- } \\
\text { co-3-hydroxyvalerate) } \\
\text { (PHBV) }\end{array}$ & - & PHBV Depolymerase & {$[28]$} \\
\hline $\begin{array}{c}\text { Bacillus sp. AF8, } \\
\text { Pseudomonas sp AF9, } \\
\text { Micrococcus sp. 10, } \\
\text { Arthrobacter sp. AF11, and } \\
\text { Corynebacterium sp. AF12 }\end{array}$ & $\begin{array}{c}\text { Soil from plastic deposition } \\
\text { sites }\end{array}$ & $\begin{array}{l}\text { Poly [4,4'-methylenebis } \\
\text { (phenyl isocyanate)-alt- } \\
\text { 1,4-butanediol/poly } \\
\text { (butylene adipate)] } \\
\text { (Polyurethane, PU) }\end{array}$ & $\begin{array}{l}\text { p-nitrophenol } \\
\text { (Spectroscopy), } \mathrm{CO}_{2} \\
\text { (Sturm test) }\end{array}$ & $\begin{array}{c}\text { Esterase } \\
\text { Polyurethanases (plate } \\
\text { assay with Coomassie } \\
\text { blue R 250). }\end{array}$ & [29] \\
\hline $\begin{array}{c}\text { PN24 Bacillus cereus, PN12 } \\
\text { Bacillus pumilus, LNR3 } \\
\text { Arthrobacter. }\end{array}$ & $\begin{array}{c}\text { Waste deposition sites and } \\
\text { artificially developed soil } \\
\text { beds containing maleic } \\
\text { anhydride } \\
\text { glucose, and small pieces } \\
\text { of polyethylene. }\end{array}$ & $\begin{array}{l}\text { High and low-density } \\
\text { polyethylenes (HDPE/ } \\
\text { LDPE) }\end{array}$ & - & - & [46] \\
\hline
\end{tabular}


Table 2. Marine, Freshwater and coastal environments

\begin{tabular}{|c|c|c|c|c|c|}
\hline ID & Habitat & Plastic type degraded & Degradation by-products & Enzyme & Reference \\
\hline $\begin{array}{c}\text { Pseudomonas, } \\
\text { Staphyloccoccus, Moraxella, } \\
\text { Micrococcus, Streptococcus }\end{array}$ & Mangrove soil & $\begin{array}{l}\text { Polyethylene bags and } \\
\text { plastic cups }\end{array}$ & 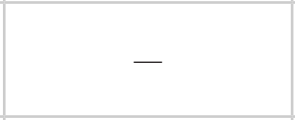 & - & [47] \\
\hline $\begin{array}{c}\text { Pseudomonas (Pseudomonas } \\
\text { stutzeri) }\end{array}$ & River water & $\begin{array}{l}\text { High molecular weight } \\
\text { Polyethylene Glycols } \\
\text { (PEG's) }\end{array}$ & Glyoxylic acid & $\begin{array}{c}\text { PEG } \\
\text { dehydrogenase } \\
\text { (Single } \\
\text { polypeptide) }\end{array}$ & {$[22]$} \\
\hline $\begin{array}{c}\text { Rhodobacteraceae, } \\
\text { Rhodospirillaceae, } \\
\text { Oceanospirillaceae, } \\
\text { Glaciecola }\end{array}$ & Seawater & $\begin{array}{c}\text { Poly(3-hydroxybutyrate- } \\
\text { co-3hydroxyhexanoate) } \\
\text { (PHBH) }\end{array}$ & 一 & - & {$[48]$} \\
\hline $\begin{array}{c}\text { Shenawella (CT01), Moritella } \\
\text { (CT12, JT01, JT04), } \\
\text { Psychrobacter (JT05) and } \\
\text { Pseudomonas (JT08) }\end{array}$ & Marine dephts soil & $\begin{array}{l}\text { Poly } \varepsilon \text {-caprolactone } \\
\text { (PCL), aliphatic } \\
\text { polyesters }\end{array}$ & - & - & [49] \\
\hline $\begin{array}{c}\text { Terrabacter tumescens, } \\
\text { Terracoccus luteus, } \\
\text { Brevibacillus reuszeri, } \\
\text { Agrobacterium tumefaciens, } \\
\text { Burkholderia vietnamiensis, } \\
\text { Duganella zoogloeoides, } \\
\text { Pseudomonas lemoignei, } \\
\text { Ralstonia eutropha, Ralstonia } \\
\text { pickettii, Matsuebacter } \\
\text { chitosanotabidus, Roseateles } \\
\text { depolymerans, Rhodoferax } \\
\text { fermentans, Variovorax } \\
\text { paradoxus, Serratia } \\
\text { marcescens, Acinetobacter } \\
\text { calcoaceticus, Acinetobacter } \\
\text { junii, Pseudomonas } \\
\text { pavonaceae, Pseudomonas } \\
\text { rhodesiae, Pseudomonas } \\
\text { amygdali, Pseudomonas } \\
\text { veronii }\end{array}$ & $\begin{array}{l}\text { Soil samples (ando-soil, } \\
\text { woody area at Shima- } \\
\text { Tsakuba, brown lowlands } \\
\text { soils, sandy riverside soil and } \\
\text { riverside mud) }\end{array}$ & $\begin{array}{c}\text { Poly }(\beta- \\
\text { hydroxyalkanoate), } \\
\text { poly }(\varepsilon- \\
\text { caprolactone), } \\
\text { poly(hexamethylene } \\
\text { carbonate), or } \\
\text { poly(tetramethylene } \\
\text { succinate) }\end{array}$ & 一 & 一 & {$[50]$} \\
\hline Bacillus mojavensis TH309 & $\begin{array}{c}\text { Bio-deteriorated plastic waste } \\
\text { from Tidal zone on Carsamba } \\
\text { coast of Samsun }\end{array}$ & $\begin{array}{l}\text { Poly(e-caprolactone) } \\
\text { (PCL) }\end{array}$ & - & Esterase (BmEST) & [51] \\
\hline $\begin{array}{l}\text { Alcalinovorax, Hyphomonas } \\
\text { and Cycloclasticus. }\end{array}$ & Seawater and soil samples & $\begin{array}{c}\text { Poly(ethylene } \\
\text { terephthalate) (PET) \& } \\
\text { Biodegradable Plastic } \\
\text { bags (BD) }\end{array}$ & $\begin{array}{l}\text { Insoluble by-products } \\
\text { of the hydrolytic } \\
\text { degradation of PET. }\end{array}$ & Esterase & {$[52]$} \\
\hline $\begin{array}{c}\text { Pseudomonas pachastrellae } \\
\text { JCM12285 }\end{array}$ & $\begin{array}{l}\text { Marine plastic debris in } \\
\text { coastal seawater }\end{array}$ & $\begin{array}{c}\text { Poly(E-caprolactone) } \\
\text { (PCL) }\end{array}$ & - & PCL hydrolase & {$[53]$} \\
\hline $\begin{array}{c}\text { Gammaproteobacteria, } \\
\text { Alphaproteobacteria, and } \\
\text { Flavobacteria (Class level) }\end{array}$ & $\begin{array}{c}\text { Microplastics exposed to } \\
\text { seawater from coastal zones }\end{array}$ & $\begin{array}{c}\text { Polypropylene (PP) and } \\
\text { polyvinyl chloride (PVC) } \\
\text { microplastics }\end{array}$ & - & - & [54] \\
\hline Arcobacter and Colwellia & $\begin{array}{c}\text { Three types of coastal marine } \\
\text { sediment from Spurn Point, } \\
\text { Humber Estuary, U.K. }\end{array}$ & $\begin{array}{l}\text { Low Density } \\
\text { Polyethylene (LDPE) } \\
\text { microplastics }\end{array}$ & - & - & {$[55]$} \\
\hline $\begin{array}{c}\text { Bacillus cereus and Bacillus } \\
\text { gottheilii }\end{array}$ & $\begin{array}{l}\text { Sediments from Matang } \\
\text { mangrove in Perak }\end{array}$ & $\begin{array}{c}\text { Polyethylene } \\
(\mathrm{PE}), \text { polyethylene } \\
\text { terephthalate (PET), } \\
\text { polypropylene (PP), and } \\
\text { polystyrene (PS) }\end{array}$ & - & - & {$[56]$} \\
\hline $\begin{array}{c}\text { Lysinibacillus fusiformis strain } \\
\text { VASB14/WL and Bacillus } \\
\text { cereus strain VASB1/TS }\end{array}$ & $\begin{array}{c}\text { Rhizosphere samples from } \\
\text { mangrove (Aviccenia marina) } \\
\text { soil }\end{array}$ & Polythene & - & - & [57] \\
\hline
\end{tabular}


Microbial research has focused on new sources. One of the most popular in recent years is the gut microbiome from organisms such as Coleoptera, Lepidoptera, Lumbricidae and certain mollusks. Some organisms contain bacterial strains capable of degrading complex chemical structured materials such as wax or wood timbers. Assays on these organisms and their gut microbiota have shown a certain capacity of degrading plastic samples as well (Table 3). These host organisms could also be referred to as holobionts ${ }^{[58]}$.

\section{General Process of Biodegradation by Microorganisms}

For a better comprehension of the topic, a deeper understanding of the biodegradation process is required. Thus this metabolic mechanism is the core of the activity and eventual application of plastic degradation through bacterial strains.

The biodegradation process may differ depending on the genera and species, but the main process is illustrated in Figure 1 and described as follows:

The general microbial biodegradation process can be described in three stages: Biodeterioration, biofragmentation and assimilation ${ }^{[66]}$. At the time environmental abiotic degradation occurs vias Mechanical, Chemical, Photocatalytic Thermal and Ozone-induced degradation ${ }^{[67]}$.

\section{Biodeterioration}

It all begins when the plastic material is exposed to the environment (where abiotic degradation factors join the process), then, bacteria start to settle down into the plastic surface to form a biofilm or the so called "Plastisphere" [68]. Microbial activity of consortia (i.e. protein and enzymatic activity) causes deterioration of physical, mechanical and chemical properties of the polymer, leading to cracking of surfaces, formation of oligomers, monomers, as well as

Table 3. Macrofauna gut microbiome (Holobionts)

\begin{tabular}{|c|c|c|c|c|c|}
\hline ID & Habitat & Plastic type degraded & Degradation by-products & Enzyme & Reference \\
\hline $\begin{array}{c}\text { Escherichia, Shigella, Asaia and } \\
\text { Acinetobacter,Rhodocytophaga, } \\
\text { Bergeyella, Diaphorobacter, } \\
\text { Hydrogenophaga, } \\
\text { Zhizhongheella. }\end{array}$ & $\begin{array}{l}\text { Gut microbiome from } \\
\text { Galleria mellonella }\end{array}$ & $\begin{array}{c}\text { Low-density } \\
\text { polyethylene (LDPE) }\end{array}$ & Glycol & - & [59] \\
\hline $\begin{array}{l}\text { Enterobacteriaceae, } \\
\text { Spiroplasmataceae, and } \\
\text { Enterococcaceae }\end{array}$ & $\begin{array}{l}\text { Gut microbes from } T \text {. } \\
\text { obscurus and T. molitor } \\
\text { (purchased from insect } \\
\text { breeding plants) }\end{array}$ & Polystyrene (PS) & - & - & [60] \\
\hline $\begin{array}{c}\text { Dyella, Lysobacter, and } \\
\text { Leptothrix }\end{array}$ & $\begin{array}{l}\text { Microbes from larvae } \\
\text { T. molitor gut. larvae } \\
\text { purchased from market }\end{array}$ & $\begin{array}{l}\text { Polystyrene (PS) and } \\
\text { Low-density } \\
\text { polyethylene (LDPE) } \\
\text { Foams }\end{array}$ & - & - & [61] \\
\hline $\begin{array}{c}\text { Family Enterobacteriaceae, } \\
\text { Sphingobacteriaceae, and } \\
\text { Aeromonadaceae }\end{array}$ & $\begin{array}{l}\text { Microbiome gut from Soil } \\
\text { Snail } A \text {. fulica purchased } \\
\text { from Jiaxing Hong- } \\
\text { Fu Breeding farm } \\
\text { (Zhejiang, China), }\end{array}$ & $\begin{array}{l}\text { Expanded polystyrene } \\
\text { (PS) foam }\end{array}$ & - & - & [62] \\
\hline Serratia sp. strain WSW & $\begin{array}{c}\text { Microflora from } P . \text { davidis } \\
\text { larvae gut }\end{array}$ & $\begin{array}{l}\text { Polystyrene (PS) } \\
\text { Styrofoam }\end{array}$ & - & - & [63] \\
\hline $\begin{array}{l}\text { Actinobacteria (Microbacterium } \\
\text { awajiense, Rhodococcus jostii, } \\
\text { Mycobacterium vanbaalenii and } \\
\text { Streptomyces fulvissimus) and } \\
\text { Firmicutes (Bacillus simplex } \\
\text { and Bacillus sp.) }\end{array}$ & Earthworm's gut & LDPE & $\begin{array}{l}\text { Volatile compounds } \\
\text { (octadecane, eicosane, } \\
\text { docosane and tricosane) } \\
\text { and nanoplastics }\end{array}$ & & [64] \\
\hline Bacillus and Serratia & $\begin{array}{c}\text { Microbial gut from } G \text {. } \\
\text { mellonella } L \text {. larvae } \\
\text { purchased from Huiyude } \\
\text { Co. }\end{array}$ & $\begin{array}{l}\text { Polyethylene (PE) and } \\
\text { polystyrene (PS) }\end{array}$ & $\begin{array}{l}\text { Long chain fatty acids as } \\
\text { the metabolic intermediates } \\
\text { of plastics in the residual } \\
\text { polymers }\end{array}$ & - & [65] \\
\hline
\end{tabular}


carbon and nitrogen sources ${ }^{[66]}$.

\section{Biofragmentation}

In order to reach polymer assimilation, microorganisms have to break polymer bonds for cellular absorption of oligomers and monomers, since polymer chemical structure is too big and complex to be directly absorbed by microorganisms ${ }^{[69]}$. This goal is reached through the secretion of polymer-specific enzymes and free radical generation ${ }^{[66]}$. Action of extracellular enzymes on a polymer is generally defined as the concept of depolymerization ${ }^{[70]}$. Biofragmentation could lead to microplastic formation if the plastic media is not assimilated yet by microorganisms ${ }^{[64]}$.

\section{Assimilation}

This stage is defined for the real absorption of the plastic atoms into the microbial cell; providing essential needs such as energy, electrons and elementary sources like carbon, nitrogen and phosphorus. Microorganisms are able to sustain and reproduce at the time they produce energy via aerobic respiration, anaerobic respiration or fermentation ${ }^{[6]]}$.

As a result of polymer cleavage, monomer/oligomer absorption and metabolic processation; microbes can release mineral molecules, contributing to natural biogeochemical processes, as well as organic molecules, which some could be ecotoxic threats under certain conditions and degrees ${ }^{[6]}$. Mineralization differs by the presence of carbon dioxide and water under aerobic conditions, and methane and carbon dioxide for anaerobic ${ }^{[67]}$.

Some byproducts laid by microbial metabolic activity through the biodegradation process could have potential use for other technological or industrial uses and applications ${ }^{[71]}$.

The chemical reactions resulting from plastic biodegradation through aerobic and anaerobic respiration are illustrated by Equation 1 and Equation 2 .

C plastic $+\mathrm{O}_{2} \rightarrow \mathrm{CO}_{2}+\mathrm{H}_{2} \mathrm{O}+\mathrm{C}$ residual + Biomass

Equation 1. Aerobic microbial biodegradation of plastic $^{[72]}$.

$\mathrm{C}$ plastic $\rightarrow \mathrm{CH}_{4}+\mathrm{CO}_{2}+\mathrm{H}_{2} \mathrm{O}+\mathrm{C}$ residual + Biomass

Equation 2. Anaerobic microbial biodegradation of plastic $^{[72]}$.

Aerobic microbial biodegradation of plastics (Equation 1 ) is performed by the use of oxygen as electron acceptor, breaking down organic chemicals into smaller organic compounds or monomers. Carbon dioxide and water are excreted as byproducts of the cleavage. Meanwhile, in anaerobic microbial biodegradation (Equation 2), microbes set nitrate, sulphate, iron, manganese and carbon dioxide as electron acceptors due to lack of oxygen for the cleavage and formation of smaller compounds ${ }^{[72]}$.

All these stages of the general biodegradation process of plastic through microbial activity are illustrated in Figure 1.

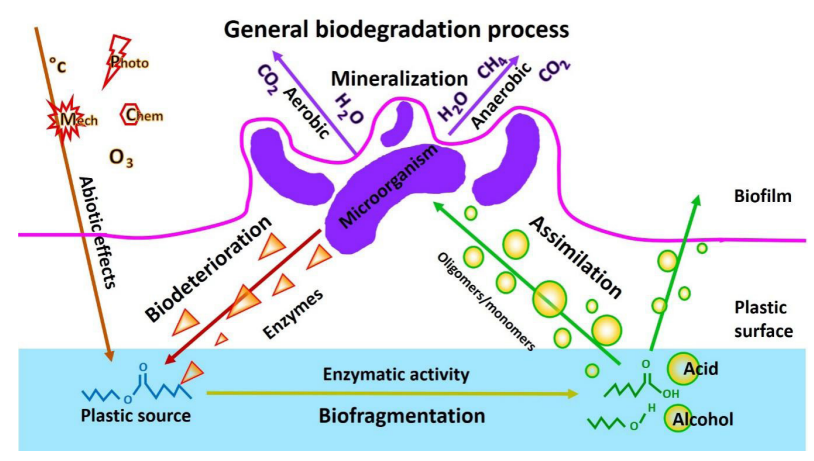

Figure 1. General biodegradation process by microorganisms. The three stages of biodeterioration are illustrated as detailed previously in this review. Note that the Chem, Mech, Photo, ${ }^{\circ} \mathrm{C}$ and $\mathrm{O}_{3}$ symbols represent Chemical degradation, Mechanical degradation, photodegradation, temperature and Ozone, as they are abiotic drivers for plastic degradation on the environment.

\section{Conclusions}

Some remarkable features about the state of the art is that bacterial strains come mostly from the bias to the human environment, rather than natural ecosystems. Waste and trash disposal sites, recycling sites, city soil samples and laboratory or purchased strains are the most common origins for plastic degrading bacteria. Otherwise, if natural environments are also well covered, most research papers focus on marine environments, mainly in seawater exposure experiments, leaving a great research gap and an opportunity for studies on coastal and wetland environments, considering that these ecosystems have great biodiversity.

Microbial biodegradation of plastic is known to be an environmentally friendly, cheap and acceptable way for plastic waste treatment ${ }^{[29]}$, so waste management actions should pay some attention to these potential opportunities.

This knowledge could be applied into technological developments for bioremediation or biomitigation of plastic polluted ecosystems. As well as integrate to municipal waste management plans, which even today are not well designed nor applied to most urban and rural locations.

Incursions into new strategies and solutions to plastic pollution will cause positive impacts on the world's ecosystems and human population, with the participation of every government level, as well as corporations and non-governmental organizations. Some other positive impacts of development and action on this subject rely on the achievement of United Nation's 2030 Sustainable Development Goals. In such objectives as Good Health and well-being, clean water and sanitation, climate action, life below water, life on land and partnerships for the goals. Industrial companies could benefit from economic incen- 
tives, positive publicity and product mark-up as a result of extending their value chain responsibility by contributing into plastic-pollution mitigation initiatives.

\section{Acknowledgements}

The research group acknowledges the PAPIIT project IT202418 for financial support, to the UMDI-Sisal and to the Faculty of Sciences of the National Autonomous University of Mexico for the facilities granted to carry out this work.

\section{Conflict of Interest}

The authors have no conflict of interest to declare.

\section{References}

[1] Cristán-Frías, A., Ize-Lema, I. and Gavilán-García, A. (2003) La situación de los envases de plástico en México Gaceta Ecológica, núm. 69, octubre-diciembre, 2003, pp. 67-82. Secretaría de Medio Ambiente y Recursos Naturales, Distrito Federal, México.

[2] North, E.J. and Halden, R.U. (2013) Plastics and Environmental Health: The Road Ahead. Rev Environ Health 28: 1-8.

DOI: 10.1515/reveh-2012-0030.

[3] Rojo-Nieto, E. and Montoto, T. (2017) "Basuras marinas, plásticos y microplásticos: orígenes, impactos y consecuencias de una amenaza global" Ecologistas en Acción ISBN:978-84-946151-9-1.

[4] NOAA. (2018) Plastic Fact Sheet. URL https:// marinedebris.noaa.gov/sites/default/files/2018_Plastics_Fact_Sheet.pdf.

[5] Barrett, J., Chase, Z., Zhang, J., Banaszak-Holl, M.M., Willis, K., Williams, A., Hardesty, B.D. and Wilcox, C. (2020) Microplastic pollution in deep-sea sediments from the Great Australian Bight. Front Mar Sci 7: 808. DOI: 10.3389/fmars.2020.576170.

[6] Napper, I.E., Davies, B.F.R., Clifford, H., Elvin, S., Koldewey, H.J., Mayewski, P. A., Miner, K. R., Potocki M., Elmore, A.C., Gajurel, A.P., Thompson, R.C. (2020) Reaching New Heights in Plastic PollutionPreliminary Findings of Microplastics on Mount Everest. One Earth 3:621-630.

DOI: 10.1016/j.oneear.2020.10.020.

[7] Ragusa A., Svelato A., Santacroce C., Catalano P., Notarstefano V., Carnevali O., Papa F., Rongioletti M. C.A., Baiocco F., Draghi S., D'Amore E., Rinaldo D., Matta M., and Giorgini E. (2020) Plasticenta: First evidence of microplastics in human placenta. Environ Int 146: 106274.

DOI: $10.1016 /$ j.envint.2020.106274.
[8] ONU Ambiente (2018) El Estado de los Plásticos: Perspectiva del Día Mundial del Medio Ambiente 2018. URL https://www.unenvironment.org/es/resources/informe/el-estado-de-los-plasticos-perspectiva-del-dia-mundial-del-medio-ambiente-2018.

[9] INEGI, (2010) Bases de datos Promedio diario de residuos sólidos urbanos recolectados por municipio y delegación. Promedio diario de residuos sólidos urbanos recolectados según método de obtención del dato por entidad federativa URL https://www.inegi. org.mx/temas/residuos/default.html\#Tabulados.

[10] SEMARNAT (2012) Informe de la Situación del Medio Ambiente en México, Capítulo 7. Residuos. URL http://biblioteca.semarnat.gob.mx/janium/Documentos/Ciga/Libros2013/CD001623.pdf.

[11] Van Sebille, E., England, M. H., and Froyland, G. (2012) Origin, dynamics and evolution of ocean garbage patches from observed surface drifters. Environ Res Lett 7: 044040.

[12] Rios, L.M., Jones, P.R., Moore, C., and Narayan, U.V. (2010) Quantitation of persistent organic pollutants adsorbed on plastic debris from the Northern Pacific Gyre's "eastern garbage patch". Journal of Environ Monit 12: 2226-2236.

[13] Derraik, J.G.B. (2002) The pollution of the marine environment by plastic debris: a review. Mar Pollut Bull 44:842-852.

[14] Van Cauwenberghe, L., \& Janssen C.R. (2014) Microplastics in bivalves cultured for human consumption. Environmental Pollution, Volume 193, Pages 65-70, ISSN 0269-7491. https://doi.org/10.1016/ j.envpol.2014.06.010.

[15] Bennett, D., Bellinger, D. C., Birnbaum, L. S., Hertz-Picciotto, I. et al. (2016) Project TENDR: Targeting Environmental Neuro-Developmental Risks the TENDR Consensus Statement. URL https://ehp. niehs.nih.gov/doi/full/10.1289/ehp358.

[16] Constanza, R., d'Arge, R., de Groot, R., Farber, S., Grasso, M., Hannon, B., Limburg, K., Naeem, S., O'Neill, R. V., Paruelo, J., Raskin, R. G., Sutton, P, Van-den, B. M. (1998) The value of the world's ecosystem services and natural capital. Ecol Econom 25: 3-15.

[17] Schwartz, M. W., Bringham, C., Hoeksema, J. D., Lyons, K. G., Mills, M.H. and van Mantgem, P.J. (2000) Linking biodiversity to ecosystem function: implications for conservation ecology. Oecologia 122: $297-305$.

[18] de Groot, R. S., Wilson Matthew A. and Boumans Roelof M.J. (2002) A typology for the classification, description and valuation of ecosystem functions, 
goods and services. Ecol Econom 41: 393-408.

DOI: 10.1016/S0921-8009(02)00089-7.

[19] Barbier, E., Hacker, S., Kennedy, C., Koch, E., Stier, A., Silliman, B. (2011). The Value of Estuarine and Coastal Ecosystem Services. Ecological Monographs. 81 .

DOI: $10.1890 / 10-1510.1$.

[20] Liu, X., Ashforth E., Ren, B., Song, F., Dai, H., Liu, M., Wang, J., Xie, Q. and Zhang, L., (2010) Bioprospecting microbial natural product libraries from the marine environment for drug discovery. J Antibiot 63: 415-422.

DOI: $10.1038 /$ ja.2010.56.

[21] Maron, P. A., Ranjard, L., Mougel, C. and Lemanceau, P. (2007) Metaproteomics: A New Approach for Studying Functional Microbial Ecology. Microb Ecol 53: 486-493. https://doi.org/10.1007/ s00248-006-9196-8.

[22] Obradors N. and Aguilar J. (1991) Efficient Biodegradation of High-Molecular-Weight Polyethylene Glycols by Pure Cultures of Pseudomonas stutzeri. Appl Environ Microbiol 57: 2383-2388.

[23] Pometto III, A. L., Lee, B., and Johnson, K. E. (1991) Production of an Extracellular Polyethylene-Degrading Enzyme(s) by Streptomyces Species. Appl Environ Microbiol 58:731-733.

[24] Muñoz-Inostroza, S.C. (2014) Degradación de polímeros de interés industrial utilizando una mezcla de Pseudomonas aeruginosa, Cladosporium sp y Alternaria sp. (Tesis para título profesional de Tecnólogo Médico) Universidad Santo Tomás Tecnología Médica.

[25] Abou-Zeid, D.M., Müller, R.J., Deckwer, W.D. (2001) Degradation of natural and synthetic polyesters under anaerobic conditions. J Biotechnol 86:113-126.

[26] Uribe, D., Giraldo, D., Gutiérrez and Merino, F. (2010) Biodegradation of low-density polyethylene by the action of a microbial consortium isolated from a landfill, Lima, Peru. Rev Peru Biol 17: 133 - 136.

[27] Kim, M.Y., Kim, C., Moon, J., Heo, J., Jung, S.P., and Kim, J.R. (2016) Polymer Film-Based Screening and Isolation of Polylactic Acid (PLA)-Degrading Microorganisms. J Microbiol Biotechnol 27: 342349.

DOI: $10.4014 / \mathrm{jmb} .1610 .10015$.

[28] Akbar, S., Hasan, F., Nadhman, A., Khan, S., Shah, A.A. (2013) Production and Purification of Poly(3-hydroxybutyrate-co-3-hydroxyvalerate) Degrading Enzyme from Streptomyces sp. AF-111. J Polym Environ 21: 1109-1116.

DOI: $10.1007 / \mathrm{s} 10924-013-0600-4$.
[29] Shah, A.A., Hasan, F., Akhter, J.I., Hameed, A. and Ahmed, S. (2008) Degradation of polyurethane by novel bacterial consortium isolated from soil. Ann Microbiol 58: 381.

DOI: $10.1007 / \mathrm{BF} 03175532$

[30] Yoshida, S., Hiraga, K., Takehana, T., Yamaji, I., Hironao, Y., Maeda, Y., Toyohara, K., Miyamoto, K., Kimura, Y., and Oda, K. (2016) A bacterium that degrades and assimilates poly(ethylene terephthalate). Science 351: 1196-1199.

DOI: $10.1126 /$ science.aad6359

[31] Gutiérrez-Pescador, J.G. (2013) Biodegradación de polietileno de baja densidad por consorcios microbianos. (Tesis de Licenciatura). Universidad Nacional Autónoma de México, México. Retrieved from https://repositorio.unam.mx/contenidos/404040.

[32] Nakajima-Kambe, T., Onuma, F., Kimpara, N., Nakahara, T., (1995) Isolation and characterization of a bacterium which utilizes polyester polyurethane as a sole carbon and nitrogen source. FEMS Microbiol Lett 129: 39-42. DOI: 10.1111/j.1574-6968.1995.tb07554.x.

[33] Uchida, H., Nakajima-Kambe, T., Shigeno-Akutsu, Y., Nomura, N., Tokiwa, Y., Nakahara, T. (2000) Properties of a bacterium which degrades solid poly(tetramethylene succinate)-co-adipate, a biodegradable plastic. FEMS Microbiol Lett 189: 25-29.

[34] Abdullahi, M. and Saidu, B.T. (2013) Biodegradation of polythene and plastic using FADAMA soil amended with organic and inorganic fertilizer. Indian J Sci Res 4: 17-24.

[35] Hadad, D., Geresh, S. and Sivan, A. (2004) Biodegradation of polyethylene by the thermophilic bacterium Brevibacillus borstelensis. J Appl Microbiol 98: 1093-1100.

DOI: 10.1111/j.1365-2672.2005.02553.x.

[36] Orr, I. G., Hadar, Y., and Sivan, A. (2004) Colonization, biofilm formation and biodegradation of polyethylene by a strain of Rhodococcus ruber. Appl Microbiol Biotechnol 65: 97-104.

DOI: $10.1007 / \mathrm{s} 00253-004-1584-8$.

[37] Danko, A.S., Luo, M., Bagwell, Christopher, E., Brigmon, R. L., and Freedman, D.L. (2004) Involvement of Linear Plasmids in Aerobic Biodegradation of Vinyl Chloride. Appl Environ Microbiol 70: 60926097.

DOI: 10.1128/AEM.70.10.6092-6097.2004.

[38] Howard, G.T. and Blake, R. C. (1998) Growth of Pseudomonas fluorescens on a polyester-polyurethane and the purification and characterization of a polyurethanase-protease enzyme. Int Biodeterior 
Biodegrad 42: 213-220.

[39] Kleeberg, I., Hetz, C., Kroppenstedt, R.M., Müller, R.J., and Deckwer, W.D. (1998) Biodegradation of Aliphatic-Aromatic Copolyesters by Thermomonospora fusca and Other Thermophilic Compost Isolates. Appl Environ Microbiol 64: 1731-1735.

[40] Elbanna, K., Lütke-Eversloh, T., Jendrossek, D., Luftmann, H., Steinbüchel, A. (2004) Studies on the biodegradability of polythioester copolymers and homopolymers by polyhydroxyalkanoate (PHA)-degrading bacteria and PHA depolymerases. Arch Microbiol 182: 212-225.

DOI: $10.1007 / \mathrm{s} 00203-004-0715-z$.

[41] Saad, J.O. (2017) Screening of Plastic Degrading Bacteria from Dumped Soil Area. IOSR J Environ Sci Toxicol Food Technol 11: 93-98.

DOI: $10.9790 / 2402-1105029398$.

[42] Abrusci, C., Pablos, J.L., Corrales, T., López-Marín, J., Marín, I., Catalina, F. (2011) Biodegradation of photo-degraded mulching films based on polyethylenes and stearates of calcium and iron as pro-oxidant additives. Int Biodeterior Biodegradation 65: 451e459.

DOI: 10.1016/j.ibiod.2010.10.012.

[43] Fontanella, S., Bonhomme, S., Koutny, M., Husarova, L., Brusson, J. M., Courdavault, J.P., Pitteri, S., Samuel, G., Pichon, G., Lemaire, J., Delort, A.M. (2010) Comparison of the biodegradability of various polyethylene films containing pro-oxidant additives. Polym Degrad Stab 95: 1011e1021.

DOI: 10.1016/j.polymdegradstab.2010.03.009.

[44] Sriyapai, P., Chansiri, K. and Sriyapai, T. (2018) Isolation and Characterization of Polyester-Based Plastics-Degrading Bacteria from Compost Soils. Microbiology 87: 290-300.

DOI: $10.1134 / \mathrm{S} 0026261718020157$.

[45] Teeraphatpornchai, T., Nakajima-Kambe, T., Shigeno-Akutsu, Y., Nakayama, M., Nomura, N., Nakahara, T., Uchiyama H. (2003) Isolation and characterization of a bacterium that degrades various polyester-based biodegradable plastics. Biotechnol Lett 25: 23-28.

[46] Satlewal, A., Soni, R., Zaidi, M., Shouche, Y., Goel, R. (2008) Comparative biodegradation of HDPE and LDPE using an indigenously developed microbial consortium. J Microbiol Biotechnol Mar 18: 477-82. PMID: 18388465.

[47] Kathirensan, K. (2003) Polythene and Plastics-degrading microbes from the mangrove soil. Rev Biol Trop 51: 629-634.

[48] Morohoshi, T., Ogata, K., Okura, T., and Sato, S.
(2018) Molecular Characterization of the Bacterial Community in Biofilms for Degradation of Poly(3-Hydroxybutyrate-co-3-Hydroxyhexanoate) Films in Seawater. Microbes Environ 00: 000-000. DOI: 10.1264/jsme2.ME17052.

[49] Sekiguchi, T., Sato, T., Enoki, M., Kanehiro, H., Uematsu, K., and Kato, C. (2010) Isolation and characterization of biodegradable plastic degrading bacteria from deep-sea environments. JAMSTEC Rep Res Dev 11: 33-41.

DOI: 10.5918/jamstecr.11.33.

[50] Suyama, T., Tokiwa, Y., Ouichanpagdee, P., Kanagawa, T., and Kamagata, Y. (1998) Phylogenetic Affiliation of Soil Bacteria That Degrade Aliphatic Polyesters Available Commercially as Biodegradable Plastics. Appl Environ Microbiol 64: 5008 - 5011. DOI: 10.1128/AEM.64.12.5008-5011.1998.

[51] Adigüzel, A.O. (2020) Production and characterization of thermo-, halo- and solvent-stable esterase from Bacillus mojavensis TH309. Biocatal Biotransformation 38: 210-226.

DOI: $10.1080 / 10242422.2020 .1715370$.

[52] Denaro, R., Aulenta, F., Crisafi, F., Di Pippo, F., Cruz-Viggi, C., Matturro, B., Tomei, P., Smedile, F., Martinelli, A., Di Lisio, V., Venezia, and C., Rossetti S. (2020) Marine hydrocarbon-degrading bacteria breakdown poly(ethylene terephthalate) (PET). Sci Total Environ 749: 141608.

DOI: $10.1016 /$ j.scitotenv.2020.141608.

[53] Suzuki, M., Tachibana, Y., Oba, K., Takizawa, R., \& Kasuya, K. (2018) Microbial degradation of poly( $\varepsilon$-caprolactone) in a coastal environment. Polym Degrad Stab 149: 1-8.

DOI: 10.1016/j.polymdegradstab.2018.01.017.

[54] Xu, X., Wang, S., Gao, F., Li, J., Zheng, L., Sun, C., He, C., Wang, Z., \& Qu, L. (2019) Marine microplastic-associated bacterial community succession in response to geography, exposure time, and plastic type in China's coastal seawaters. Mar Pollut Bull 145: 278-286.

DOI: 10.1016/j.marpolbul.2019.05.036.

[55] Harrison, J.P., Schratzberger, M., Sapp, M. and Osborn, A.M. (2014) Rapid bacterial colonization of low-density polyethylene microplastics in coastal sediment microcosms. BMC Microbiology 14: 232.

[56] Auta, H. S., Emenike, C. U., Fauziah, S. H. (2017) Screening of Bacillus strains isolated from mangrove ecosystems in Peninsular Malaysia for microplastic degradation. Environ Pollut 231: 1552e1559.

DOI: 10.1016/j.envpol.2017.09.043.

[57] Shahnawaz, M., Sangale, M. K. and Ade, A.B. (2016) 
Rhizosphere of Avicennia marina (Forsk.) Vierh. as a landmark for polythene degrading bacteria. Environ Sci Pollut Res 23: 14621-14635.

DOI: $10.1007 / \mathrm{s} 11356-016-6542-3$.

[58] Madhusoodanan J. (2019) Do hosts and their microbes evolve as a unit? PNAS 116: 14391-14394. URL www. pnas.org/cgi/doi/10.1073/pnas.1908139116.

[59] Cassone, B. J., Grove, H. C., Elebute, O., Villanueva, S. M. P. and LeMoine, C. M. R. (2020) Role of the intestinal microbiome in low-density polyethylene degradation by caterpillar larvae of the greater wax moth, Galleria mellonella. Proc R Soc B 287: 20200112.

DOI: $10.1098 / \mathrm{rspb} .2020 .0112$.

[60] Peng, B.Y., Su, Y., Chen, Z., Chen, J., Zhou, X., Benbow, M. E., Criddle, C. S., Wu, W.M., and Zhang, Y. (2019) Biodegradation of Polystyrene by Dark (Tenebrio obscurus) and Yellow (Tenebrio molitor) Mealworms (Coleoptera: Tenebrionidae). Environ Sci Technol 53: 5256-5265.

DOI: 10.1021/acs.est.8b06963.

[61] Yang, L., Gao, J., Liu, Y., Zhuang, G., Peng, X., Wu, W.M., and Zhuang, X. (2021) Biodegradation of expanded polystyrene and low-density polyethylene foams in larvae of Tenebrio molitor Linnaeus (Coleoptera: Tenebrionidae): Broad versus limited extent depolymerization and microbe-dependence versus independence. Chemosphere 262: 127818.

DOI: 10.1016/j.chemosphere.2020.127818.

[62] Song, Y., Qiu, R., Hu, J., Li, X., Zhang, X., Chen, Y., Wu, W.M., and He, D. (2020) Biodegradation and disintegration of expanded polystyrene by land snails Achatina fulica. Sci Total Environ 746: 141289.

DOI: 10.1016/j.scitotenv.2020.141289.

[63] Woo, S., Song, I., and Cha, H. J. (2020) Fast and Facile Biodegradation of Polystyrene by the Gut Microbial Flora of Plesiophthalmus davidis Larvae. Appl Environ Microbiol 86: e01361-20.

DOI: 10.1128/AEM.01361-20.

[64] Huerta-Lwanga, E., Thapa, B., Yang, X., Gertsen, H., Salánki, T., Geissen, V., and Garbeva, P. (2017) Decay of low-density polyethylene by bacteria ex- tracted from earthworm's guts: A potential for soil restoration. Sci Total Environ 624: 753-757.

DOI: 10.1016/j.scitotenv.2017.12.144.

[65] Lou, Y., Ekaterina, P., Yang, S. S., Lu, B., Liu, B., Ren, N., Corvini, P. F. X., and Xing, D.(2020) Biodegradation of Polyethylene and Polystyrene by Greater Wax Moth Larvae (Galleria mellonella L.) and the Effect of Co-diet Supplementation on the Core Gut Microbiome. Environ Sci Technol 54: 2821-2831.

DOI: $10.1021 /$ acs.est.9b07044.

[66] Lucas, N., Bienaime, C., Belloy, C., Queneudec, M., Silvestre, F., Nava-Saucedo, J.E. (2008) Polymer biodegradation: Mechanisms and estimation techniques. Chemosphere 73: 429-442.

[67] Singh, B., and Sharma, N. (2008) Mechanistic implications of plastic degradation. Polym Degrad Stab 93: 561-584.

DOI: 10.1016/j.polymdegradstab.2007.11.008.

[68] Zettler, E. R., Mincer, T., Proskurowski, G., Amaral-Zettler, L. A. (2011) The "Plastisphere": A new and expanding habitat for marine protists. J Phycol 47: pS45-pS45.

[69] Müller, R. J. (2005) Biodegradability of Polymers: Regulations and Methods for Testing. Online, A. Steinbüchel (Ed.). DOI: $10.1002 / 3527600035$.bpola012.

[70] Glaser, J.A. (2019). Biological Degradation of Polymers in the Environment. In Plastics in the Environment. Gomiero, A. IntechOpen.

DOI: 10.5772/intechopen.85124.

[71] Savoldelli, J., Tomback, D., Savoldelli, H. (2017) Breaking down polystyrene through the application of a two-step thermal degradation and bacterial method to produce usable byproducts. Waste Management, Volume 60, ISSN 0956-053X. DOI: 10.1016/j.wasman.2016.04.017

[72] Alshehrei, F. (2017) Biodegradation of Synthetic and Natural Plastic by Microorganisms. Appl Environ Microbiol 5: 8-19.

DOI: 10.12691/jaem-5-1-2. 